\title{
Apoio computacional para a Mediação Pedagógica em "Debate de Teses"
}

\author{
Sabrina Siqueira Panceri ${ }^{1,2}$, Crediné Silva de Menezes $^{1,3}$ \\ ${ }^{1}$ Universidade Federal do Espírito Santo - Programa de Pós-Graduação em Informática \\ Av. Fernando Ferrari, 514, Goiabeiras - Vitória - ES - Brasil - Cx. Postal 29075-910 \\ ${ }^{2}$ Instituto Federal do Espírito Santo - Campus Guarapari \\ Estrada da Tartaruga, s/n, Muquiçaba - Guarapari - ES - Brasil - Cx. Postal 29215-090 \\ ${ }^{3}$ Universidade Federal do Rio Grande do Sul - Faculdade de Educação \\ Av. Paulo Gama, s/n, Farroupilha - Porto Alegre - RS - Brasil - Cx. Postal 90046-900 \\ sabrina.panceridifes.edu.br, credinedgmail.com
}

\begin{abstract}
This paper presents the development of a master's research aimed at supporting teachers in the practice of pedagogical mediations in virtual environments. We used as a basis to identify possible mediations pedagogical application of Pedagogical Architecture Debate of Theses. We developed a computational solution called ALPES to support the practice of these mediations. We implement part of this tool and apply it in a real scenario for data collection. The collected data were analyzed and the results obtained from this analysis provide evidence that the proposed solution provides grants to support the practice of pedagogical mediations in virtual environments.
\end{abstract}

Resumo. Este artigo apresenta o desenvolvimento de uma pesquisa de mestrado direcionada a apoiar o docente na prática de mediações pedagógicas em ambientes virtuais. Utilizamos como base para identificar possíveis mediações pedagógicas a aplicação da Arquitetura Pedagógica Debate de Teses. Elaboramos uma solução computacional denominada ALPES para apoiar a prática dessas mediações. Implementamos parte desta ferramenta e a aplicamos em um cenário real para coleta de dados. Os dados coletados foram analisados e os resultados obtidos com essa análise apresentam evidências que a solução proposta fornece subsídios para apoiar a prática de mediações pedagógicas em ambientes virtuais.

\section{Introdução}

Com o advento da cultura digital, novas possibilidades de interação tornaram-se possíveis. Estas novas possibilidades trazem consigo a oportunidade de um novo papel para o usuário, o de produtor de informações. Seguindo essa tendência, os ambientes virtuais utilizados para promover a Educação a Distância permitem o registro das interações realizadas entre os alunos em suas diversas formas de comunicação e troca de informações pelo ambiente.

Dentro dessa realidade, o professor pode (re)visitar os espaços e produções de cada aluno buscando informações para auxiliá-los de diversas formas. Essas novas possibilidades de interação entre alunos e professores apoiam o advento de uma nova 
V Congresso Brasileiro de Informática na Educação (CBIE 2016)

Anais dos Workshops do V Congresso Brasileiro de Informática na Educação (CBIE 2016)

forma de "fazer a educação", onde os espaços físicos e tempos definidos das aulas dão espaço às comunicações assíncronas realizadas através de dispositivos de comunicação diversos de qualquer lugar e em qualquer tempo.

Com o intuito de organizar as interações realizadas através dos ambientes virtuais de apoio ao ensino para potencializar a construção de conhecimento dos alunos Carvalho e outros (2007) propuseram o conceito de Arquiteturas Pedagógicas. Neste trabalho utilizamos uma delas, a Arquitetura Pedagógica Debate de Teses [Nevado et al. 2011], que será apresentada com mais detalhes na Seção 2.

Apresenta-se como desafio para o professor monitorar e analisar o grande volume de textos gerados durante as interações propostas pelas arquiteturas pedagógicas. Faz-se necessário a criação de suporte computacional para facilitar a recuperação das informações contidas nestes textos. Assim, esperamos facilitar o processo de análise e monitoramento das interações orientadas pela Arquitetura Pedagógica Debate de Teses e também minimizar os esforços e o tempo gasto pelo professor na prática dessas atividades.

\section{Caracterização do Problema}

A Arquitetura Pedagógica Debate de Teses (APDT) [Nevado et al. 2011] foi elaborada seguindo os preceitos Piagetianos sobre a construção de conhecimento (Piaget apud [Ramozzi-Chiarottino 1988]). Neste sentido, a APDT "busca apoiar as aprendizagens, sistematizando interações que favoreçam/incentivem esses movimentos num processo de construção conjunta do conhecimento". Concebida no contexto da cultura digital, onde os sujeitos interagem de forma assíncrona e geograficamente distribuídos com a mediação de redes de comunicação, propõe a construção de conhecimento dos participantes de forma colaborativa através de suas interações orientadas.

Nas interações, que são feitas através da produção de textos, os indivíduos expõem suas convicções a respeito de teses (afirmações) propostas pelo mediador (professor). Uma visão geral sobre a APDT com suas interações definidas como etapas é apresentada pela Figura 1.

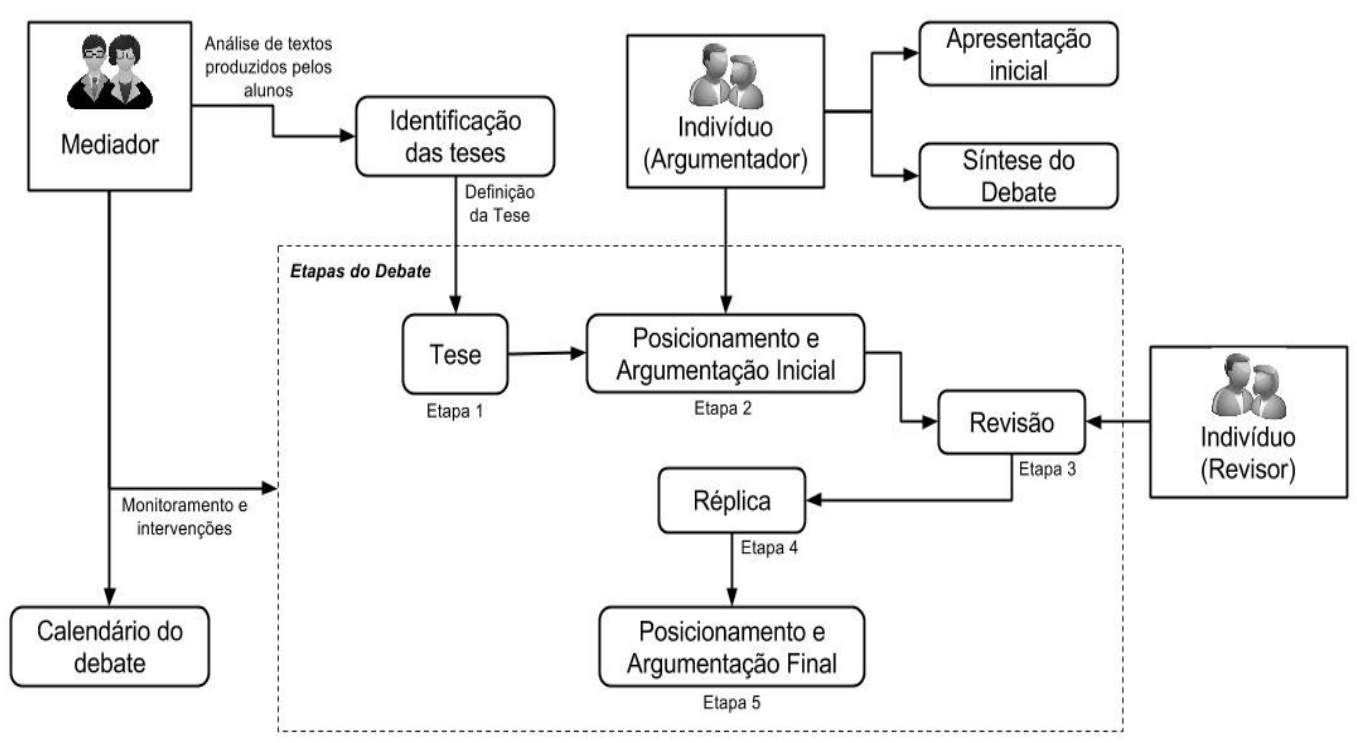

Figura 1. Visão geral da Arquitetura Pedagógica Debate de Teses 
Durante todo o desenvolvimento do debate o Mediador (professor) analisa e monitora os textos produzidos pelos alunos buscando pelos elementos requeridos de cada uma das fases ou etapas do debate. Os indivíduos (alunos) elaboram seus textos e realizam interações com outros indivíduos nas etapas 2 à 5 . As demais fases e/ou etapas feitas pelos alunos equivalem a registros de seus conhecimentos no início e término no debate.

A partir da análise das interações e do volume de textos produzidos, realizamos um estudo sobre a APDT no qual identificamos as necessidades e/ou possibilidades para a realização de mediações pedagógicas. As mediações pedagógicas elicitadas têm como objetivos: analisar, compreender e fornecer feedback para os indivíduos, e são realizadas pelo professor (mediador) visando auxiliar no processo de construção de conhecimento dos indivíduos que participam da APDT.

De forma geral, as mediações pedagógicas podem ser vistas como intervenções individuais ou coletivas. Contudo, de acordo com os preceitos das arquiteturas pedagógicas, o professor deve se ater a realizar intervenções que ajudem os indivíduos na construção de seu conhecimento e não a limitá-la. Dentre as mediações pedagógicas identificadas, destacamos as mediações "Agrupar indivíduos que possuem argumentações semelhantes" e "Elaborar síntese das argumentações finais para realizar feedback coletivo".

Empregar as mediações pedagógicas de forma manual, ou seja, com o professor acessando as produções de cada indivíduo, lendo, compreendendo e destacando características demandam um tempo relevante do professor, o que dificulta sua prática, uma vez que o professor desenvolve outras atividades além do acompanhamento desta atividade em específico. Uma forma de diminuir o tempo dedicado à aplicação das mediações pedagógicas e torná-las uma prática viável é utilizar a tecnologia como apoio para realização dessas tarefas, através da construção de ferramentas computacionais capazes de analisar e identificar características nos textos, e assim minimizar os esforços do professor.

\section{Fundamentação Teórica}

De acordo com Wooldridge e Jennings apud [Wooldridge 2009] um Agente Inteligente deve possuir, junto com a autonomia, as seguintes características: Reatividade; Proatividade; e Habilidades sociais. Reis (2003) define Sistemas Multiagente (SMA) como "um Sistema Multiagente é um sistema computacional em que dois ou mais agentes interagem ou trabalham em conjunto de forma a desempenhar determinadas tarefas ou satisfazer um conjunto de objetivos".

Segundo Coppin (2004) em muitas situações, um SMA pode ser composto apenas por agentes reativos, uma vez os agentes não existem de forma isolada e na prática, os agentes são compostos por um conjunto de regras, logo, suas ações são limitadas. Ainda assim, SMA são considerados sistemas complexos tanto para sua modelagem, quanto para seu desenvolvimento. Para isso, metodologias que apoiam a elaboração de projeto de software para SMA foram desenvolvidas.

Partindo dos modelos e notações da UML, várias linguagens e metodologias foram propostas para serem aplicadas a projetos de SMA. Wagner e Taveter apud [Guedes 2012] apresentam a AORML - Agent-Object-Relationship Modeling Language, que tem por objetivo suportar o projeto de alto nível de SMA. 
De modo geral um SMA deve ser composto por técnicas e algoritmos que serão utilizados por seus agentes para atingir os objetivos para os quais foram projetados. A seguir apresentamos a fundamentação teórica estudada sobre Recuperação de Informações em Textos, foco computacional principal de nossa pesquisa.

A Recuperação de Informações (RI) tem como foco a busca de informações em documentos ou textos e a classificação destas buscas a fim de retornar os documentos mais importantes em uma coleção, dada uma quantidade limitada de informações a serem resgatadas. De acordo com Manning e outros (2009) "RI busca encontrar material de natureza não estruturada que satisfaça uma necessidade de informação, a partir de grandes coleções". Técnicas de pré-processamento de textos são utilizadas em conjunto com técnicas de RI e servem para preparar o corpus para posterior análise. As técnicas exploradas nesta pesquisa foram: Remoção de Termos Frequentes ou Stopwords; Etiquetagem Morfossintática; Normalização; Redução ao Radical ou Stemming.

De acordo com Dumais (2004), a Análise Semântica Latente (LSA, do inglês Latent Semantic Analysis) é "uma abordagem estatística totalmente automática utilizada para extrair relações entre palavras por meio de seus contextos de uso em documentos, passagens ou frases". Além disso, a LSA é considerada uma técnica de aprendizado de máquina não supervisionado. A LSA também pode ser considerada como um modelo de representação de dados ou textos, uma vez que o resultado final de aplicação da mesma é uma matriz de representação da frequência e importância das palavras dentro de um corpus, que considera a proximidade entre duas palavras e sua possível relação semântica.

Em outro viés das pesquisas em RI encontram-se os Algoritmos de Agrupamento ou Clusterização, que são utilizados para, a partir da análise de características semelhantes entre os textos que compõem o corpus, criar grupos de semelhança onde membros de um mesmo grupo possuem um maior nível de semelhança entre eles do que com os membros de outros grupos. Segundo Manning e outros (2009), a Clusterização é a forma mais comum de aprendizado não supervisionado, ou seja, não há interferência humana no processo. O Algoritmo K-Means (KM) é um dos mais importantes algoritmos de clusterização e foi o algoritmo utilizado nesta pesquisa para realizar os agrupamentos.

\section{Trabalhos Correlatos}

Durante a elaboração desta pesquisa realizamos uma investigação na literatura científica, mais especificamente em artigos publicados em revistas e anais de eventos, entre os anos de 1992 a 2015. Realizadas as buscas, selecionamos através da leitura dos resumos dos artigos àqueles que continham técnicas e/ou conceitos que pudessem nos nortear. Essa busca resultou na catalogação de 119 artigos. Destes 119, selecionamos 40 artigos para uma análise mais detalhada. Finalizada esta análise, selecionamos 8 artigos que julgamos conter maior proximidade aos nossos objetivos de pesquisa. O trabalho mais próximo de nosso contexto de pesquisa, Debate Dashboard, foi desenvolvido por Iandoli e outros (2014) e apresentado no artigo "Socially Augmented Argumentation Tools: Rationale, Design and Evaluation of a Debate Dashboard" [Iandoli et al. 2014].

O Debate Dashboard foi desenvolvido para simular a dinâmica de um debate regular, tendo como objetivo apresentar de forma gráfica para o aluno quais foram suas contribuições no processo de debate e resgatar afirmações que possam ajudar no 
V Congresso Brasileiro de Informática na Educação (CBIE 2016)

Anais dos Workshops do V Congresso Brasileiro de Informática na Educação (CBIE 2016)

momento de fechamento do debate. $O$ foco da solução é o aluno e não o professor/mediador. Suas semelhanças com nossa proposta de solução que será apresentada na Seção 5, estão em ambas as soluções utilizarem como base de pesquisa sistemas de suporte ao processo de debate, além do uso de técnicas de Recuperação de Informações para realizar a análise dos textos produzidos durante os debates. Como diferenças principais destacamos que o Debate Dashboard tem como objetivo apresentar para o aluno como está sua participação do debate, utiliza Mapas Conceituais para apresentar de modo gráfico as afirmações que resgata a partir das discussões do debate e possui uma dinâmica de debate tradicional, o que difere da dinâmica proposta pela APDT. Além disso, nosso objetivo é criar e fornecer suporte computacional para auxiliar o professor enquanto mediador de um debate que segue os moldes da APDT.

\section{Descrição da Solução}

A partir da análise das mediações pedagógicas identificadas e com o objetivo principal de facilitar a análise das produções realizadas durante as interações da APDT, elaboramos uma solução computacional seguindo a arquitetura de Sistemas Multiagente (SMA), denominada ALPES. A partir das orientações da Linguagem AORML, que utiliza o Diagrama de Casos de Uso (DCU) com a abstração dos agentes através de fronteiras, elaboramos o diagrama que representa o ALPES e o apresentamos na Figura 2.

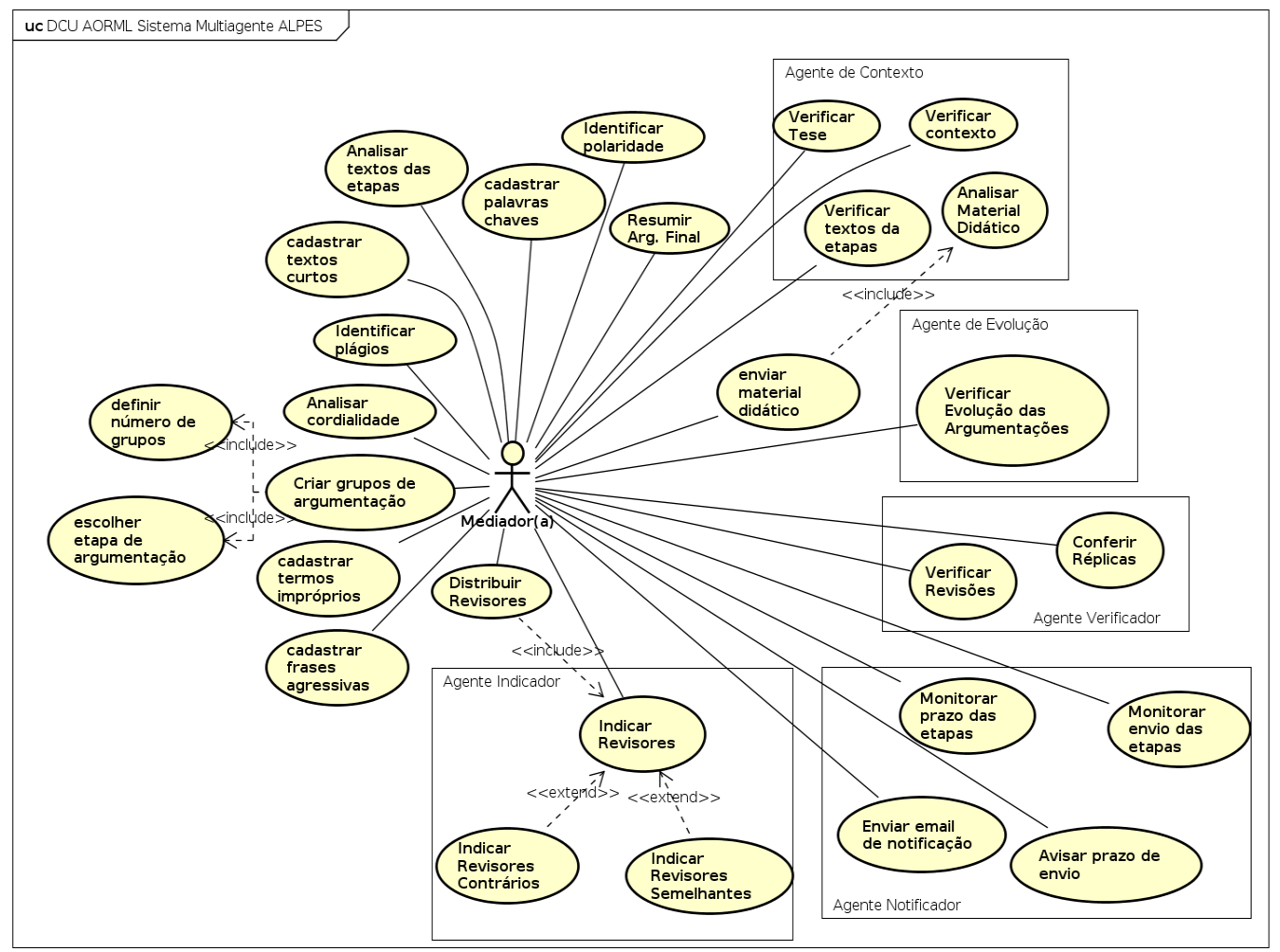

Figura 2. Diagrama de Casos de Uso AORML Sistema Multiagente ALPES

Os agentes do SMA ALPES são reativos, logo, a partir de alterações em suas áreas de influência os agentes são acionados. Apresentamos em sequência a descrição dos cinco agentes que compõem o SMA ALPES.

1. Agente Verificador: Foi projetado para monitorar o envio das etapas de Revisão e Réplica, a fim de verificar se os textos produzidos nestas etapas 
V Congresso Brasileiro de Informática na Educação (CBIE 2016)

Anais dos Workshops do V Congresso Brasileiro de Informática na Educação (CBIE 2016)

estão de acordo com os objetivos da respectiva etapa.

2. Agente de Evolução: Este agente é responsável por comparar os textos produzidos na etapa de Argumentação Inicial com os demais textos das demais etapas, a busca de elementos que foram acrescentados na Argumentação Final.

3. Agente Indicador: Este agente foi projetado para agilizar o processo de distribuição de revisores. Seu fluxo de ações é iniciado pelo Recurso Indicação de Revisores.

4. Agente Notificador: Responsável por avisar os alunos que ainda não enviaram seus textos os prazos de cada etapa do debate, utilizando como base o calendário definido pelo mediador ao configurar o debate no Sistema "Debate de Teses".

5. Agente de Contexto: Inicia suas ações no momento em que um aluno realiza o salvamento ou envio do texto de qualquer uma etapa. Este agente monitora todas as etapas, a fim de auxiliar o aluno a produzir seus textos seguindo o tema proposto pela tese.

Apresentamos a seguir um resumo sobre os recursos disponíveis no ALPES.

1. Grupos de Similaridade: Este recurso está diretamente vinculado à mediação pedagógica "Agrupar indivíduos que possuem argumentações semelhantes". Apresenta como resultado a criação de subgrupos de acordo com a semelhança entre os textos produzidos.

2. Identificação de "similaridades" entre textos: A partir da análise da Argumentação Final, verifica se existem trechos de textos idênticos produzidos por alunos diferentes e apresenta um relatório com esses destaques para o professor.

3. Indicação de Revisores: De acordo com critérios definidos pelo professor, realiza a distribuição de revisores.

4. Análise de cordialidade: Este recurso está relacionado a mediação "Verificar se houve quebra de cordialidade nas revisões e réplicas". Analisa as revisões e réplicas em busca de termos ou expressões impróprias.

5. Identificação de polaridade: Verificar se o argumento inicial ou final está de acordo com o posicionamento indicado.

6. Síntese das autorias: Apresenta um resumo das argumentações finais produzidas por um grupo de alunos.

\subsection{Núcleo de Recuperação de Informações}

O Núcleo de Recuperação de Informações (NRI) foi elaborado usando técnicas, métodos e algoritmos apresentados na Seção 3. O NRI foi construído como um conjunto de pacotes, onde cada pacote contém classes que são responsáveis por analisar e processar os textos a fim de recuperar informações relevantes, dada uma determinada solicitação (entrada).

Na Figura 3 mostramos o Diagrama de Pacotes do NRI. Nela temos os quatro pacotes internos: Processamento Textual, Modelos de Representação, Análise de Similaridade e Clusterização. O pacote Processamento Textual possui quatro pacotes internos: Remoções, Etiquetagem, Stemming e Normalização. O pacote Modelos de Representação contém dois pacotes internos: LSA e SVM. Além disso, estão

1 Sistema de apoio à APDT. Disponível em: http://www.pead.faced.ufrgs.br/sites/cms/debate/ 
V Congresso Brasileiro de Informática na Educação (CBIE 2016)

Anais dos Workshops do V Congresso Brasileiro de Informática na Educação (CBIE 2016)

exemplificadas as relações de dependência entre os pacotes.

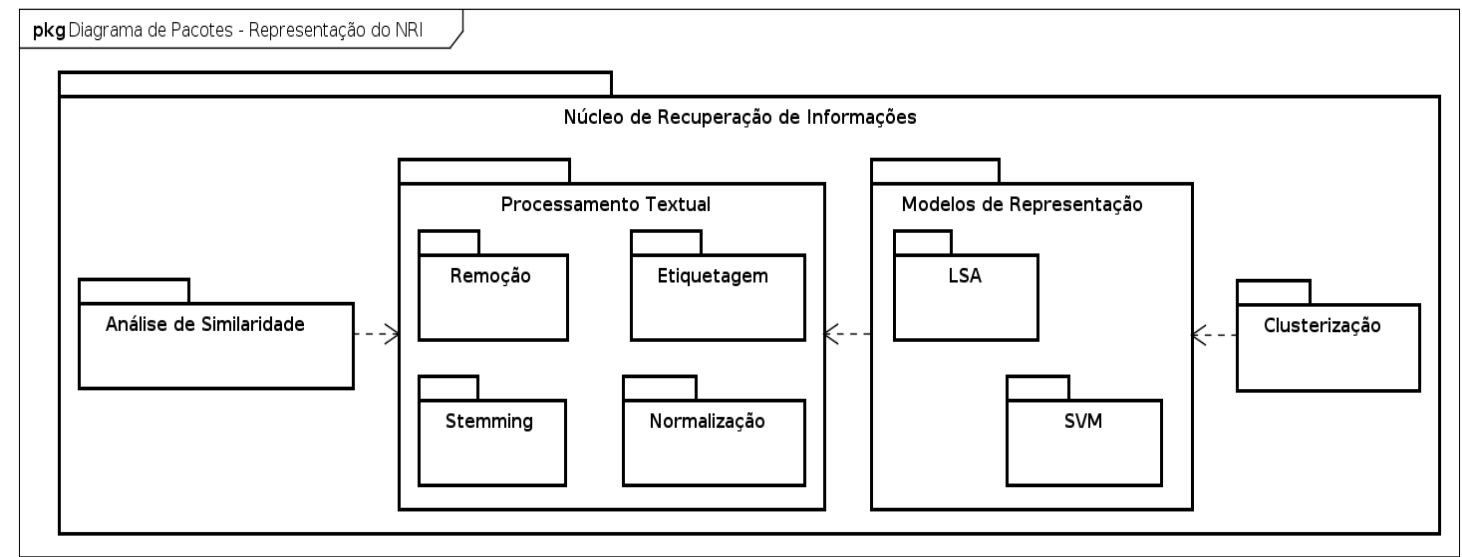

Figura 3. Diagrama de Pacotes - Representação do Núcleo de Recuperação de Informações

Objetivo computacional principal desta pesquisa, o NRI foi concebido para processar os textos, e a partir desse processamento dar suporte aos agentes e demais recursos do sistema. Assim, para comprovar que os recursos disponibilizados pelo SMA ALPES mostram-se como uma solução favorável para apoiar o Mediador, sistematizamos uma prova de conceito que segue descrita na Seção 6.

\section{Prova de Conceito}

$\mathrm{Na}$ condução deste estudo foram implementadas todas as funcionalidades do Núcleo de Recuperação de Informações (NRI) e os recursos Grupos de Similaridade e Síntese das Autorias. O recurso Grupos de Similaridade foi escolhido pois, para sua perfeita execução é necessário que todas as funcionalidades do NRI também fossem implementadas. Além disso, a criação manual de grupos de alunos mostrou-se como uma das tarefas mais custosas em relação ao tempo do mediador, o que nos motivou a comparar os resultados alcançados pela solução aos resultados obtidos de forma manual.

Já o recurso Síntese das Autorias foi escolhido por utilizar parte do NRI para sua execução e porque essa atividade necessita que o mediador leia várias vezes os textos dos alunos para conseguir destacar os termos que ele considere como mais importantes.

\subsection{Protótipo Computacional}

O protótipo computacional é um sistema web, desenvolvido com base no Framework Django 1.6 [Django 2014] e na linguagem de programação Python 2.7.6 [Python 2014]. Para garantirmos que o sistema pode ser acessado em dispositivos diversos que possuam um navegador web e acesso à internet, as interfaces de acesso foram desenvolvidas a partir de modelos disponibilizadas pelo Projeto Bootstrap ${ }^{2}$. Por ser um sistema web, o desenvolvimento do protótipo segue a Arquitetura Cliente-Servidor.

$\mathrm{Na}$ Figura 4 apresentamos uma abstração do protótipo seguindo esse modelo de arquitetura. Na camada Cliente temos a interação de acesso entre Mediador e o ALPES, o envio de informações entre o ALPES e o Sistema "Debate de Teses". Na camada Servidor temos os componentes de ambos sistemas, sendo que os componentes do Alpes

2 Mais informações em http://getbootstrap.com/about/ 
V Congresso Brasileiro de Informática na Educação (CBIE 2016)

Anais dos Workshops do V Congresso Brasileiro de Informática na Educação (CBIE 2016)

são seu banco de dados, o Núcleo de Recuperação de Informações (NRI) e os Agentes. Já o SDT tem seu banco de dados.

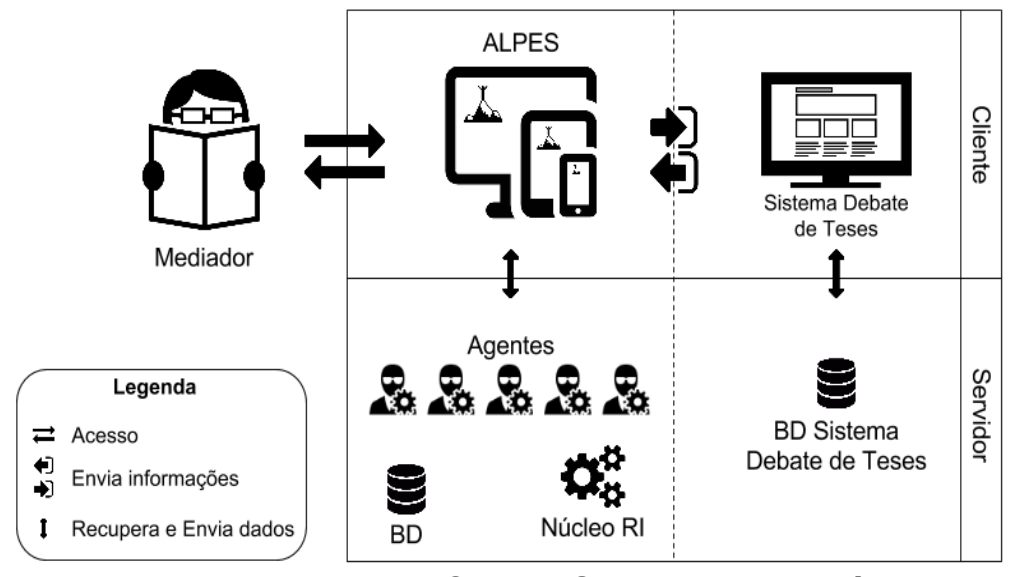

Figura 4. Arquitetura Cliente-Servidor do Protótipo

Os recursos disponíveis nessa versão do protótipo fazem uso de todas as funcionalidades do NRI e são descritos a seguir:

- Recurso Grupos de Similaridade: Para seu funcionamento é necessário que o mediador informe quantos grupos deseja que sejam criados e qual argumentação será utilizada como base para a criação dos grupos, ou seja, o mediador deve escolher entre a argumentação inicial e argumentação final. Após realizadas as escolhas, o mediador deve clicar no botão "Criar grupos" para iniciar o processo do recurso. Em seguida, o sistema resgatará os textos da argumentação escolhida do banco de dados do Sistema "Debate de Teses" (SDT), irá processá-los com o uso das técnicas de limpeza de corpus e técnicas de pré-processamento, criará a representação dos textos com os modelos de representação e, por fim, utilizará a clusterização para criar os grupos. Finalmente, os grupos são apresentados ao mediador.

- Recurso Síntese das Autorias: Apresenta ao mediador os termos mais importantes escritos pelos alunos na fase de Argumentação Final. Os textos das argumentações finais são recuperados do banco de dados do SDT, sobre os quais aplicam-se técnicas para limpeza dos textos, técnicas de préprocessamento, SVM como modelo de representação dos textos e a clusterização para criação de grupos de semelhança. Após a criação dos grupos, aplicamos a medida TF-IDF nas argumentações finais dos alunos que fazem parte de um mesmo grupo para identificar quais os termos mais relevantes. Os termos identificados são apresentados na forma de nuvem para o mediador.

\section{Aplicação da prova de conceito a um "Debate de Teses"}

Com o objetivo de comprovarmos a utilização da prova de conceito desenvolvida como facilitadora do processo de mediação pedagógica, comparamos o processo de criação manual de grupos com a aplicação do recurso Grupos de Similaridade, ambos a partir da análise da argumentação inicial produzida pelos alunos. Os textos analisados foram produzidos com a aplicação da APDT como atividade prévia ao curso de extensão XIV Maratona de Empreendedorismo da UFGRS realizado em 2013 [Michels 2014]. A amostra foi composta pelos 21 alunos que concluíram todas as etapas propostas pela 
APDT, e os agrupamentos foram realizadas sobre as argumentações iniciais produzidas sobre a tese "O mundo mudou, o perfil do consumidor mudou, e, consequentemente, a forma de se planejar negócios também mudou".

$\mathrm{O}$ experimento foi dividido em três fases. Na primeira fase (Fase 1) realizamos a divisão manual dos grupos através da leitura da argumentação inicial de cada aluno. $\mathrm{Na}$ segunda fase (Fase 2) utilizamos o recurso Grupos de Similaridade do protótipo computacional para criar os grupos a partir da análise automática dos textos. Na última fase do experimento (Fase 3) comparamos os grupos criados na Fase $1 \mathrm{com}$ os grupos criados na Fase 2. Por fim, apresentamos a análise dos resultados alcançados.

Algumas comparações são inevitáveis, como o tempo gasto na Fase 1 e Fase 2 do experimento. Na Fase 1 demoramos cerca de $3 \mathrm{~h}$ para criar os grupos de forma manual. Já na Fase 2 os grupos foram criados em menos de 10 min pelo recurso. Destacamos que os grupos criados na Fase 1 possuem critérios de distribuição distintos dos grupos criados na Fase 2, pois ao analisarmos os textos utilizamos nossos conhecimentos prévios sobre o contexto em que os textos foram escritos fazendo uma análise semântica dos mesmos. Já com a aplicação do recurso a análise realizada leva em consideração a semelhança sintática entre os textos, baseado no valor de semelhança encontrado com a aplicação das métricas e algoritmos que compõem o NRI e que são utilizados pelo recurso. Apesar de seguirem critérios distintos durante seus processos de criação, obtivemos um total de $75 \%$ de semelhança entre os grupos formados na Fase 1 comparados aos grupos formados na Fase 2.

Desta maneira entendemos que o experimento atesta a eficácia de uso do recurso e evidencia uma nova possibilidade para tratar as informações produzidas pelos alunos em suas interações, com o intuito de facilitar a aplicação das Mediações Pedagógicas em Ambientes Virtuais de Ensino e Aprendizagem.

\section{Conclusões}

Com o experimento realizado e descrito na Seção 7 concluímos que ao utilizar recursos computacionais para agilizar a execução de algumas atividades, o professor poderá utilizar seu tempo e esforço em outras tarefas que necessitem de sua atenção. Dessa forma, confirmamos que o uso de suporte computacional é uma boa alternativa para apoiar as atividades do professor em ambientes virtuais de aprendizagem. Ainda com apoio do experimento conseguimos confirmar, parcialmente, que o uso desse suporte computacional viabiliza o desenvolvimento de mediações pedagógicas.

A partir da concepção do Núcleo de Recuperação de Informações conseguimos demonstrar que o processamento de textos baseado na sintaxe foi suficiente para destacar semelhanças entre textos e com isso fornecer informações que facilitem o emprego de mediações pedagógicas em ambientes virtuais. Parte central da versão atual do protótipo, o núcleo desenvolvido foi capaz de correlacionar os textos produzidos pelos alunos e agrupá-los a partir da análise de semelhança entre eles.

Ao revisitarmos a concepção da proposta de solução, o desenvolvimento do protótipo e a condução do experimento, bem como a análise dos resultados obtidos, concluímos que conseguimos demonstrar que o uso de suporte computacional pode ajudar o professor na execução de suas atividades, reduzindo seu esforço e tempo despendido com as mesmas. Além disso, o suporte computacional resultante desta pesquisa de mestrado, possibilita o desenvolvimento de novas estratégias pedagógicas e 
V Congresso Brasileiro de Informática na Educação (CBIE 2016)

Anais dos Workshops do V Congresso Brasileiro de Informática na Educação (CBIE 2016)

sua aplicação em conjunto às arquiteturas pedagógicas diversas e também pode auxiliar na prática das mediações pedagógicas conduzidas em ambientes virtuais de ensino e aprendizagem.

Como trabalhos futuros, destacamos como proposta de melhoria para a versão descrita nesta pesquisa, a incorporação de técnicas de Processamento de Linguagem Natural que deem suporte à análise semântica dos textos, com aporte em ontologias de domínio que podem ser predefinidas no sistema ou que poderão ser construídas pelo professor através de um módulo específico. Por fim, seria interessante criar uma abstração do núcleo de processamento de informações para apoiar a mediação pedagógica em conjunto com as demais arquiteturas pedagógicas que utilizam a produção de textos e conversações como base para suas interações.

\section{Referências}

Carvalho, Marie Jane S.; Nevado, Rosane A.; Menezes, Crediné S. de. Arquiteturas pedagógicas para educação a distância. Aprendizagem em rede na educação a distância: estudos e recursos para formação de professores. Porto Alegre: Ricardo Lenz, v. 1, p. 36-52, 2007.

Coppin, Ben. Artificial intelligence illuminated. Jones \& Bartlett Learning, 2004.

Django1.6. 2014. Disponível em $<$ https://www.djangoproject.com/ $>$. Acesso em $10 / 01 / 2016$.

Dumais, Susan T. Latent semantic analysis. Annual review of information science and technology, v. 38, n. 1, p. 188-230, 2004.

Guedes, Gilleanes T. A. Um Metamodelo UML para a Modelagem de Requisitos em Projetos de Sistemas MultiAgentes. 2012. Tese de Doutorado, Universidade Federal do Rio Grande do Sul, Porto Alegre.

Iandoli, Luca et al. Socially augmented argumentation tools: Rationale, design and evaluation of a Debate Dashboard. International Journal of Human-Computer Studies, v. 72, n. 3, p. 298-319, 2014.

Manning, C. D.; Raghavan, P.; Schutze, H. An Introduction to Information Retrieval. Cambridge University Press. Edição Online. 2009. Disponível em: $<$ http://www.informationretrieval.org/>. Acesso em: 20/10/2015

Michels, A. B. (2014) Do Fazer ao Compreender no Contexto da Educação a Distância: Uso de Arquiteturas Pedagógicas no Processo de Empreender. Dissertação de Mestrado, Universidade Federal do Rio Grande do Sul, Porto Alegre.

Nevado, R. A. et al. (2011) Debate de Teses - Uma Arquitetura Pedagógica. Em: Anais do $22^{\circ}$ Simpósio Brasileiro de Informática na Educação, volume 1, páginas 820-829.

Python 2.7.6. 2014. Disponível em $<$ https://docs.python.org/2.7/about.html $>$ Acesso em 10/01/2016.

Ramozzi-Chiarottino, Z. (1988) Psicologia e epistemologia genética de Jean Piaget. Epu.

Reis, Luís Paulo. Coordenação em Sistemas Multi-Agente: Aplicações na Gestão Universitária e Futebol Robótico. 2003. Tese de Doutorado, Faculdade de Engenharia da Universidade do Porto. Portugal.

Wooldridge, M. (2009) An Introduction To Multiagent Systems. John Wiley \& Sons. 